Konrad Isernhagen ${ }^{1}$ Eckart Struck ${ }^{2}$

\section{Schlafqualität und Tagesschläfrigkeit bei substituierten drogenabhängigen Patienten}

Eine anonymisierte bundesweite Fragebogenstudie

\author{
Sleep Quality and Daytime Sleepiness in Drug Addicts Receiving Opioid \\ Maintenance Therapy - Anonymous Questionnaire Based Nationwide Study \\ in the Federal Republic of Germany.
}

\section{Zusammenfassung}

Wir führten eine Fragebogenstudie zu Schlafstörungen bei substituierten Drogenabhängigen an 34 Substitutionspraxen durch; 597 Patienten wurden eingeschlossen. Der Pittsburgh Sleep Quality Index und die Epworth Sleepiness Scale wurden eingesetzt. Substitutionspatienten mit Ausnahme von DHC weisen eine signifikant schlechtere Schlafqualität auf. Patienten unter Naltrexon unterscheiden sich nicht signifikant von der Kontrollgruppe, Buprenorphinpatienten zeigen eine Tendenz zu besserer Schlafqualität im Vergleich zu Methadon/Levomethadon. Die Medikamentendosis war positiv mit schlechterer Schlafqualität korreliert. Somit müssen pharmakologische Effekte als Ursache für Schlafstörungen in Betracht gezogen werden. Kontrollierte prospektive Studien sind notwendig, insbesondere die Ergebnisse für DHC sowie die Hinweise für bessere Schlafqualität unter Buprenorphin sollten weiter verfolgt werden.

\section{Schliuisselwörter}

Schlafqualität · Schlafstörung · Tagesschläfrigkeit · Methadon · Drogenabhängigkeit · Opioidsubstitutionstherapie

\section{Abstract}

Sleep disturbances in patients receiving opioid maintenance therapy (OMT) is an often observed phenomenon.There are few data about the frequency and the circumstances of sleep disturbance among these patients. Therefore we performed a study using the Pittsburgh Sleep Quality Index and the Epworth Sleepiness Scale. 597 patients and 104 controls were evaluated. Patients under OMT had a significant worse sleep quality excepting patients receiving dihydrocodeine and former drug addicts under naltrexone. Patients receiving buprenorphine showed a tendency towards better sleep quality compared to those receiving methadone/levomethadone. Consumption of alcohol and dosage of medication positively correlated with impaired sleep quality. We conclude, that pharmacologic effects, caused by opioid medication, may be partly responsible for sleep disturbance. Prospective and controlled studies are needed, especially to define the role of dihydrocodeine and buprenorphine in sleep disturbed opiate addicts.

Key words

Sleep quality · sleep disturbance · daytime sleepiness · methadone $\cdot$ drug addiction - opioid maintenance therapy

\section{Einleitung}

Im unselektierten Patientengut einer Allgemeinpraxis sind Schlafstörungen ein häufiges Phänomen. Angehörige von Berufsgruppen, die mit Suchtpatienten befasst sind, sind nach ihrem subjektiven Eindruck verstärkt mit Klagen über Schlafstörungen konfrontiert. Daten, die diesen subjektiven Eindruck bestätigen oder widerlegen, liegen nicht vor.
Die Instrumente der Schlafmedizin wie Schlafpolygraphie und der Multiple Schlaflatenz Test (MSLT) sind aufwändig und daher für größere Studien mit einer repräsentativen Teilnehmerzahl nicht geeignet. Studien mit großen Fallzahlen greifen daher oft auf Selbstbeurteilungsfragebogen zurück. 
Die Beurteilung der Schlafqualität durch den Patienten ist subjektiv. Differenzen zwischen den Ergebnissen der Selbsteinschätzung der Schlafqualität durch diverse standardisierte Fragebogen und objektiver Befunderhebung durch die Schlafpolygraphie sind gut untersucht. Die Schlaflatenz, Total sleep time, und die Schlafeffektivität werden bei Patienten mit primärer Insomnie regelmäßig schlechter eingeschätzt, es besteht aber eine hochsignifikante Korrelation zwischen den Fragebogenscores und den Ergebnissen der Schlafpolygraphie, die eine Vorhersage der Laborergebnisse durch die Fragebögen zulassen [1, 2]. Die Häufigkeit nächtlichen Erwachens wird unterschätzt [2].

Zur Messung der Tagesschläfrigkeit wird die Epworth Sleepiness scale (ESS) [3] genutzt. Es besteht eine signifikant negative Korrelation zwischen ESS score und den Resultaten des MSLT [3, 4, 5, 6].

Angaben über die Prävalenz von Schlafstörungen schwanken erheblich. In drei deutschen Studien betrug sie 19 - 25\% [7, 8, 9].

Größere Studien zu Schlafstörungen bei substituierten Drogenabhängigen liegen bisher nicht vor. Eine Untersuchung mit standardisierten Schlaffragebogen an 27 Methadonpatienten [10] zeigte eine erniedrigte TST, eine verlängerte Schlaflatenz und eine nicht signifikante Tendenz zu verminderter Schlafeffektivität.

Zahlreiche Faktoren sind geeignet, bei Drogenabhängigen Schlafstörungen zu verursachen:

- der von der Droge geprägte Lebensstil und zahllose weitere psychosoziale Faktoren

- Depression und andere psychiatrische/psychosomatische Erkrankungen, z. B. Panik-Störungen [11]

- Alkohol [12,13]

- Benzodiazepine $[12,14]$

- körperliche Erkrankungen, z. B. HIV Infektion [15]

Auch von Opiaten selbst - einschließlich Methadon - sind Veränderungen der Schlafparameter bekannt (s. Tab.1). Typisch für Patienten mit Insomnie ist eine vermehrte Tagesmüdigkeit; eine verstärkte Einschlaftendenz am Tage (Hypersomnie) findet sich nur bei Patienten mit umschriebenen organischen, Schlafstörungen z. B. Narkolepsie oder obstruktives Schlafapnoe-Syndrom [9]. Es gibt Hinweise, dass Tagesschläfrigkeit kaum von den in der Schlafpolygraphie erhobenen Parametern wie Schlaflatenz, Schlafeffektivität oder Total sleep time abhängt mit Ausnahme der Anzahl der Arousals. Schlafunterbrechungen führen bei gleicher TST zu signifikanter Verkürzung der Einschlaflatenz im Multiplen-Schlaflatenz-Test [25]. Bei Stepanski [26] wies nur die Gruppe mit exzessiver Tagesschläfrigkeit eine signifikante Korrelation zwischen MSLT-Messungen und der Anzahl der Arousals auf. Auch zu diesem Thema gibt es keine größeren Untersuchungen bei substituierten Drogenabhängigen. Chronisch gestörter Schlaf und vermehrte Tagesmüdigkeit können bei Drogenpatienten zu Depressivität und Rückfällen führen [15]. Es besteht daher Bedarf, Daten über die Schlafqualität bei einer größeren Gruppe von substituierten Drogenabhängigen zu erheben. Die hier vorliegende Studie soll klären, ob

1. Unterschiede in der Schlafqualität und in der Tagesschläfrigkeit von substituierten Drogenabhängigen im Vergleich zur Kontrollgruppe und zu ehemaligen Drogenabhängigen (unter Naltrexon) bestehen
2. Unterschiede in o. g. Parametern zwischen den einzelnen Substitutionsmedikamenten bestehen

3. eine Abhängigkeit der Schlafqualität und der Tagesschläfrigkeit von den erhobenen biografischen und anamnestischen Daten besteht.

\section{Material und Methoden}

Es wurden im gesamten Bundesgebiet Praxen, die Substitutionsbehandlungen durchführen, angeschrieben und um Mitarbeit gebeten.

34 Praxen (darunter LKH Köln-Merheim als einzige Institutsambulanz - in dieser Studie nur für Naltrexonpatienten) haben sich an der Studie beteiligt.

Die Praxen wurden gebeten, den Bedarf an Fragebogen bei der Studienleitung anzufordern.

992 Fragebogen wurden ausgegeben, davon wurden 512 Bogen ausgefüllt zurückgesandt (Rücklaufquote 51,6\%)

5 Praxen haben die Bogen selbst kopiert, daher gibt es keine sicheren Angaben über deren Rücklauf; diese Praxen haben 111 ausgefüllte Fragebogen beigesteuert.

Somit ergibt sich folgendes Gesamtbild:

$\begin{array}{lr}\text { Rücklauf gesamt } & 623 \\ \text { nicht auswertbar } & 22 \\ \text { Orlaampatienten } & 4 \\ \text { ausgewertet } & 597\end{array}$

Die Gruppenzuordnung erfolgte nach dem zum Erhebungszeitpunkt verabreichten Substitutionsmedikament, die Kontrollgruppe wurde alters- und geschlechtsadaptiert aus Patienten der Praxis des Studienleiters gewonnen.

Wegen der Berichte über bedrohliche Herzrhythmusstörungen unter Orlaam ${ }^{\circledR}$ und der nachfolgenden Marktrücknahme zu Studienbeginn konnte diese Gruppe nicht weiter ausgebaut werden und wurde in die Auswertung nicht einbezogen.

\section{Fragebogen}

Den Patienten wurde ein dreiteiliger Fragebogen mit der Bitte um Ausfüllen ohne Namensnennung vorgelegt. Im ersten Teil werden biografische und anamnestische Daten erfragt:

Alter, Geschlecht, Zeitpunkt des ersten Heroinkonsums, Dauer der Substitution, aktuelle Dosis, Alkoholkonsum und Dauermedikation neben dem Substitutionsmedikament.

Der zweite Teil besteht aus dem Pittsburgh-Sleep-Quality-Index (PSQI) [27], übersetzt und variiert vom Autor. Aus 19 Items werden sieben Komponenten (Teilscores) gebildet, die wiederum zu 
Tab. 1 Opiatwirkungen auf den Schlaf

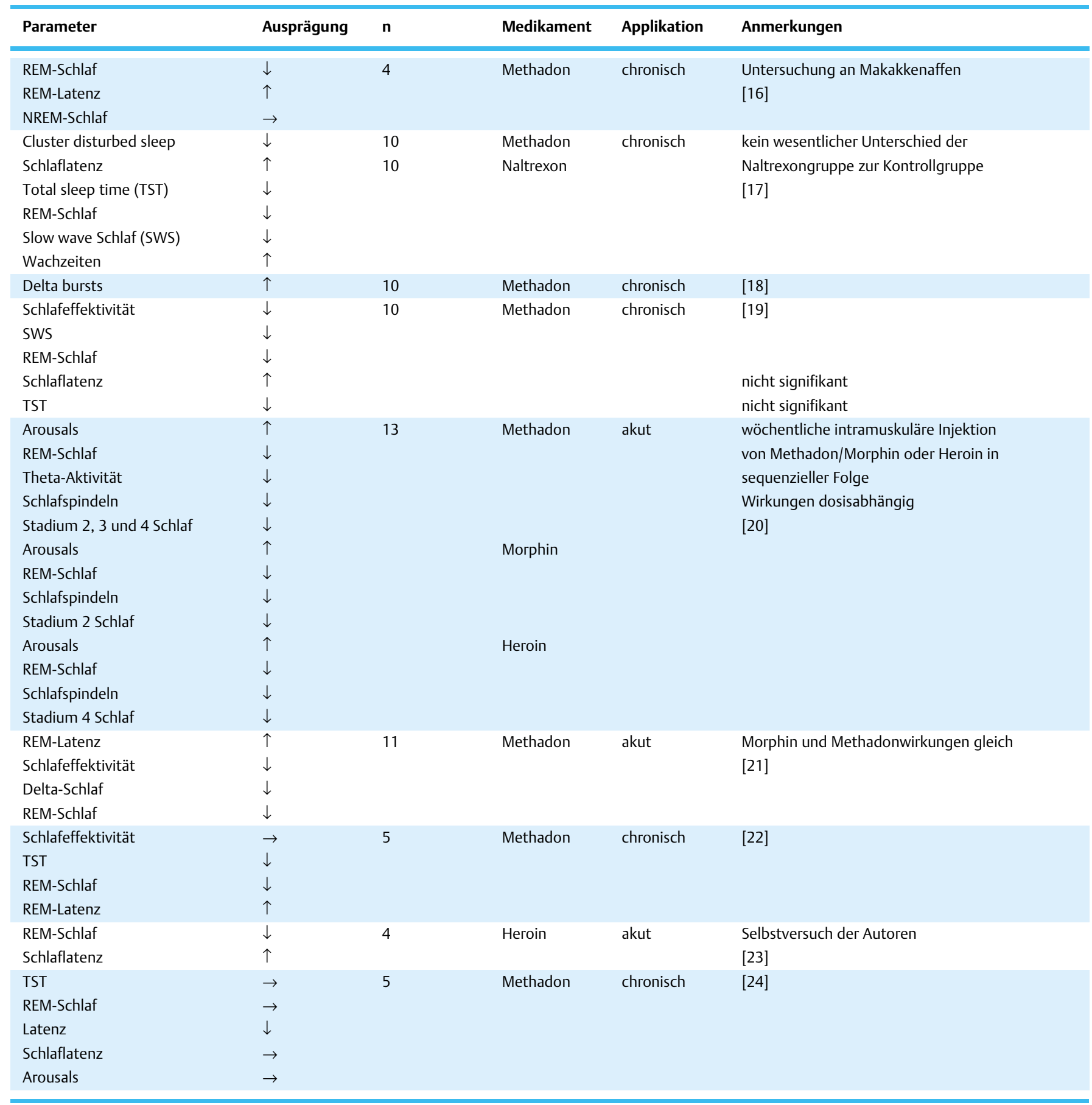

einem Globalscore zusammengefasst werden. Die sieben Teilscores können einen Wert zwischen 0 (überhaupt kein Problem) bis 3 (ein sehr großes Problem) annehmen. Damit kann der Globalscore zwischen 0 und 21 liegen, ein Wert über 5 bedeutet eine im Vergleich zu Schlafgesunden beeinträchtigte Schlafqualität.

Den dritten Teil des Fragebogens bildet die Epworth sleepiness scale (ESS) [3].

Die ESS besteht aus acht Fragen zu Alltagssituationen, in denen die Wahrscheinlichkeit einzuschlafen auf einer Skala von 0 - 3 eingeschätzt werden soll, wobei 3 die höchste Wahrscheinlich- keit einzuschlafen ausdrückt. Ein maximaler Score von 24 ist somit zu erreichen.

\section{Statistische Analyse}

Für die Untersuchung von Mittelwertunterschieden zwischen den untersuchten Personengruppen wurden t-Tests für voneinander unabhängige Stichproben verwendet (es wurde die zweiseitige Null-Hypothese $H_{0}: \mu_{1}=\mu_{2}$ bei $\alpha=5 \%$ getestet). Varianzgleichheit als Voraussetzung für den t-Test wurde mit dem Levene-Test untersucht. Im Fall, dass der Levene-Test die Hypo- 
these der Varianzgleichheit abwies, wurde der t-Test mit getrennten Varianzen berechnet. Diese Tests sind in den Tabellen ausgewiesen.

Die Differenz der Anteilswerte wurde mit einem Test auf Differenz der Anteilswerte gerechnet. Der entsprechende Test auf Ordinaleskalenniveau kam in allen Fällen zum gleichen Ergebnis wie der Test auf Anteilswerte.

Einfaktorielle Varianzanalysen wurden eingesetzt, um Aussagen über Abhängigkeiten des PSQI von Faktoren wie Alter oder Geschlecht zu machen.

Kronbachs $\alpha$ als Maß für die interne Konsistenz des PSQI ergab einen Wert von 0,80 .

\section{Ergebnisse}

\section{Gruppencharakteristika}

Die Gruppencharakteristika sind in Tab. 2 dargelegt. Nicht alle Patienten haben Angaben zu Alter, Geschlecht oder Daten der Abhängigkeit gemacht. Das Alter und die Abhängigkeitsdauer der Patienten in der DHC-Gruppe ist am höchsten, ansonsten sind die Daten vergleichbar.

\section{PSQI-Globalscore}

Tab. 3 zeigt die Ergebnisse des PSQI-Globalscores im Vergleich der Kontrollgruppe zu den Behandlungsgruppen und den Vergleich der Behandlungsgruppen untereinander. Methadon-, Levomethadon- und Buprenorphinpatienten haben einen signifikant höheren Score, DHC-Patienten haben ebenfalls einen höheren Score, der Unterschied ist jedoch nicht signifikant, Naltrexonpatienten weisen einen geringen, ebenfalls nicht signifikanten Unterschied zur Kontrollgruppe auf.

Im Vergleich der einzelnen Behandlungsgruppen zeigt Naltrexon gegenüber Methadon/Levomethadon einen signifikant niedrigeren Score, der Unterschied zwischen Naltrexon und DHC bzw.
Buprenorphin ist nicht signifikant. Die Methadon- und Levomethadongruppe haben signifikant höhere Scores im Vergleich zu DHC, im Vergleich zu Buprenorphin wird keine Signifikanz erreicht.

\section{Durchschlafstörungen}

Frage 5b des PSQI bezieht sich auf Durchschlafstörungen. Alle Behandlungsgruppen außer Naltrexon weisen signifikant höhere Scores im Vergleich zur Kontrollgruppe auf, die Differenz von DHC zur Kontrollgruppe ist geringer ausgeprägt (Tab. 4).

\section{Nächtliches Wasserlassen}

Auf Schlafstörungen infolge nächtlichen Wasserlassens wird in Frage $5 c$ des PSQI eingegangen.

Sämtliche Behandlungsgruppen außer Naltrexon erreichen signifikant höhere Scores im Vergleich zur Kontrollgruppe, Naltrexon hat zu Methadon und Levomethadon signifikant niedrigere Scores, zu DHC und Buprenorphin wird keine Signifikanz erreicht.

Methadon und Levomethadon haben zu Buprenorphin signifikant höhere scores (Tab. 5).

Es bestand ein signifikanter Einfluss zwischen der Angabe von regelmäßigem Alkoholgenuss und Schlafstörungen durch nächtliches Wasserlassen.

\section{Anteil schwerer Schlafstörungen (PSQI-Globalscore > 10)}

Der Anteil schwerer Schlafstörungen, definiert als PSQI-Globalscore $>10$, wurde mittels Test auf Differenz der Anteilswerte ermittelt und ergab für Kontrollen (8,7\% Anteil) gegen Methadon (39\%), Levomethadon (37,6\%) und Buprenorphin (37,5\% signifikante Unterschiede auf $\mathrm{p}=0,01$. Die Differenzen zu DHC (20\%) und Naltrexon (23,5\%) erreichen keine Signifikanz.

Tab. 2 Gruppencharakteristika

\begin{tabular}{|c|c|c|c|c|c|c|c|c|c|c|c|}
\hline \multirow[t]{2}{*}{ Gruppe } & \multicolumn{3}{|c|}{ Geschlecht } & \multirow{2}{*}{$\begin{array}{l}\text { Alter } \\
\text { mean } \\
\text { min - max }\end{array}$} & \multicolumn{3}{|c|}{ Abhängigkeitsdauer (Jahre) ${ }^{1)}$} & \multicolumn{3}{|c|}{ Substitutionsdauer (Jahre) } & \multirow{2}{*}{$\begin{array}{l}\text { Dosis in mg } \\
\text { mean } \\
\text { min - max }\end{array}$} \\
\hline & $n$ & $m$ & $w$ & & mean & $\min$ & $\max$ & mean & $\min$ & $\max$ & \\
\hline dl -Methadon & $\begin{array}{l}395 \\
56,3 \%\end{array}$ & $\begin{array}{l}268 \\
68,0 \%\end{array}$ & $\begin{array}{l}126 \\
32,0 \%\end{array}$ & $\begin{array}{l}34 \\
18-58\end{array}$ & 14 & 1,5 & 33 & 3,9 & 0,08 & 20 & $\begin{array}{l}78 \\
5-250\end{array}$ \\
\hline Levomethadon (Polamidon ${ }^{\circledR}$ ) & $\begin{array}{l}101 \\
14,4 \%\end{array}$ & $\begin{array}{c}59 \\
59,0 \%\end{array}$ & $\begin{array}{c}41 \\
41,0 \%\end{array}$ & $\begin{array}{l}38 \\
21-51\end{array}$ & 18,3 & 3 & 34 & 6,5 & 0,5 & 22 & $\begin{array}{l}55,1 \\
2,5-170\end{array}$ \\
\hline Buprenorphin (Subutex ${ }^{\circledR}$ ) & $\begin{array}{c}64 \\
9,1 \%\end{array}$ & $\begin{array}{c}41 \\
65,1 \%\end{array}$ & $\begin{array}{c}22 \\
34,9 \%\end{array}$ & $\begin{array}{l}32,8 \\
19-58\end{array}$ & 10,5 & 1 & 26 & 2,4 & 0,04 & 14 & $\begin{array}{c}8,5 \\
1,2-20\end{array}$ \\
\hline Dihydrocodein ${ }^{2}$ & $\begin{array}{c}20 \\
2,9 \%\end{array}$ & $\begin{array}{c}14 \\
70 \%\end{array}$ & $\begin{array}{c}6 \\
30 \%\end{array}$ & $\begin{array}{l}42,2 \\
36-73\end{array}$ & 20,5 & 7 & 33 & 7,2 & 4 & 16 & \\
\hline Naltrexon (Nemexin ${ }^{\circledR}$ ) & $\begin{array}{c}17 \\
2,4 \%\end{array}$ & $\begin{array}{c}16 \\
94,1 \%\end{array}$ & $\begin{array}{c}1 \\
5,9 \%\end{array}$ & $\begin{array}{l}31,5 \\
19-48\end{array}$ & 10,2 & 1,5 & 28 & 0,76 & 0,17 & 3 & \\
\hline Kontrollen & $\begin{array}{l}104 \\
14,8 \%\end{array}$ & $\begin{array}{c}64 \\
61,5 \%\end{array}$ & $\begin{array}{c}40 \\
38,5 \%\end{array}$ & $\begin{array}{l}33,2 \\
19-50\end{array}$ & & & & & & & \\
\hline
\end{tabular}

\footnotetext{
1) gerechnet vom ersten Zeitpunkt des Heroinkonsums, evtl. Cleanzeiten wurden nicht berücksichtigt

2) keine Dosisangabe, da zu ungenaue Angaben in den Bogen
} 
Tab. 3 PSQI-Globalscore: t-Tests

\begin{tabular}{|c|c|c|c|c|c|c|}
\hline \multicolumn{7}{|c|}{ Kontrollen gegen Behandlungsgruppen } \\
\hline & \multicolumn{6}{|c|}{ t-Test } \\
\hline & n & Mean & SD & $\mathbf{t}$ & df & $\mathbf{p}$ \\
\hline Kontrollen mit ... & 104 & 5,58 & 3,37 & & & \\
\hline Levomethadon & 101 & 10,05 & 4,96 & $-7,53^{1)}$ & df 175,7 & $<0,01$ \\
\hline Buprenorphin & 64 & 8,25 & 4,30 & $-5,03^{1)}$ & df 110,10 & $<0,01$ \\
\hline \multicolumn{7}{|c|}{ Naltrexon gegen andere Behandlungsgruppen } \\
\hline & \multicolumn{6}{|c|}{ t-Test } \\
\hline & $\mathbf{n}$ & Mean & SD & $\mathbf{t}$ & df & $\mathbf{p}$ \\
\hline Naltrexon mit ... & 17 & 6,94 & 5,02 & & & \\
\hline Methadon & 395 & 9,45 & 4,68 & $-2,15$ & df 410 & 0,032 \\
\hline & \multicolumn{6}{|c|}{ t-Test } \\
\hline & $\mathbf{n}$ & Mean & SD & $\mathbf{t}$ & df & $\mathbf{p}$ \\
\hline Methadon mit ... & 395 & 9,45 & 4,68 & & & \\
\hline Buprenorphin & 64 & 8,25 & 4,30 & 1,16 & df 457 & n. s. \\
\hline $\mathrm{DHC}$ & 20 & 7,15 & 4,83 & 2,14 & df 413 & 0,033 \\
\hline Levomethadon mit... & 101 & 10,05 & 4,96 & & & \\
\hline Buprenorphin & 64 & 8,25 & 4,30 & 1,73 & df 163 & n. s. \\
\hline $\mathrm{DHC}$ & 20 & 7,15 & 4,83 & 2,40 & df 119 & 0,018 \\
\hline
\end{tabular}

t-Test mit ungleichen Varianzen

Tab. 4 t-Tests Frage 5b PSQI (Durchschlafstörungen)

\begin{tabular}{|c|c|c|c|c|c|c|}
\hline \multicolumn{7}{|c|}{ Kontrollgruppe gegen Behandlungsgruppen } \\
\hline & \multicolumn{6}{|c|}{ t-Test } \\
\hline & n & Mean & SD & $\mathbf{t}$ & df & $\mathbf{p}$ \\
\hline Kontrollen mit ... & 104 & 1,26 & 1,05 & & & \\
\hline Methadon & 395 & 2,20 & 1,05 & $-8,11$ & df 497 & $<0,01$ \\
\hline Levomethadon & 101 & 2,27 & 0,98 & $-7,10$ & df 203 & $<0,01$ \\
\hline Buprenorphin & 64 & 2,11 & 1,17 & $-4,87$ & df 168 & $<0,01$ \\
\hline $\mathrm{DHC}$ & 20 & 1,85 & 1,14 & $-2,27$ & df 122 & 0,025 \\
\hline Naltrexon & 17 & 1,65 & 1,37 & $-1,17^{1)}$ & df 19,2 & n. s. \\
\hline
\end{tabular}

t-Test mit ungleichen Varianzen

\section{Tagesschläfrigkeit}

Die Tagesschläfrigkeit, gemessen durch die Epworth sleepiness scale, zeigt keine signifikanten Differenzen zwischen Behandlungsgruppen und Kontrollgruppen. Lediglich die Naltrexongruppe zeigt eine signifikant niedrigere Tagesschläfrigkeit im Vergleich zur Kontrollgruppe ( $p=0,01$ ).

\section{Korrelation PSQI/ESS}

Die Untersuchung auf Korrelationen zwischen PSQI-Globalscore und ESS score (Pearsons r) erbrachte für die Kontrollgruppe, die Levomethadongruppe, die DHC- und Buprenorphingruppe keine Signifikanzen. In der Methadongruppe fand sich eine positive
Korrelation $(p=0,002)$ in der Naltrexongruppe eine negative Korrelation $(\mathrm{p}=0,026)$.

\section{Einflüsse von Durchschlafstörungen (Frage 5b PSQI) und Schlafeffektivität (Komponente 4 PSQI) auf den ESS score} In keiner Gruppe konnte ein Einfluss von Durchschlafstörungen auf den ESS score nachgewiesen werden.

Ebensowenig zeigte sich ein Zusammenhang zwischen Schlafeffektivität und dem ESS score. 
Tab. 5 t-Tests Frage 5c PSQI (nächtliches Wasserlassen)

\begin{tabular}{|c|c|c|c|c|c|c|}
\hline \multicolumn{7}{|c|}{ Kontrollgruppe gegen Behandlungsgruppen } \\
\hline & \multicolumn{6}{|c|}{ t-Test } \\
\hline & n & Mean & SD & $\mathbf{t}$ & df & $\mathbf{p}$ \\
\hline Kontrollen mit ... & 104 & 0,93 & 1,05 & & & \\
\hline Methadon & 395 & 1,91 & 1,07 & $-8,28$ & df 497 & $<0,01$ \\
\hline Levomethadon & 101 & 2,03 & 1,04 & $-7,49$ & df 203 & $<0,01$ \\
\hline Buprenorphin & 64 & 1,59 & 1,12 & $-3,81$ & df 166 & $<0,01$ \\
\hline DHC & 20 & 1,65 & 1,18 & $-2,73$ & df 122 & $<0,01$ \\
\hline Naltrexon & 17 & 1,24 & 1,03 & $-1,10$ & df 119 & n. s. \\
\hline \multicolumn{7}{|c|}{ Naltrexon gegen andere Behandlungsgruppen } \\
\hline & \multicolumn{6}{|c|}{ t-Test } \\
\hline & $\mathrm{n}$ & Mean & SD & $\mathbf{t}$ & df & $\mathbf{p}$ \\
\hline Naltrexon mit ... & 17 & 1,24 & 1,03 & & & \\
\hline Methadon & 395 & 1,91 & 1,07 & $-2,53$ & df 410 & 0,012 \\
\hline Levomethadon & 101 & 2,03 & 1,04 & $-2,91$ & df 116 & 0,004 \\
\hline Buprenorphin & 64 & 1,59 & 1,12 & $-1,17$ & df 79 & n.s. \\
\hline $\mathrm{DHC}$ & 20 & 1,65 & 1,18 & $-1,13$ & df 79 & n. s. \\
\hline \multicolumn{7}{|c|}{ Methadon und Levomethadon gegen andere Behandlungsgruppen } \\
\hline & \multicolumn{6}{|c|}{ t-Test } \\
\hline & $\mathbf{n}$ & Mean & SD & $\mathbf{t}$ & df & $\mathbf{p}$ \\
\hline Methadon mit ... & 395 & 1,91 & 1,07 & & & \\
\hline Buprenorphin & 64 & 1,59 & 1,15 & $2,04^{1)}$ & df 81,65 & 0,045 \\
\hline Levomethadon mit ... & 101 & 2,03 & 1,04 & & & \\
\hline Buprenorphin & 64 & 1,59 & 1,15 & 2,46 & df 124,47 & 0,015 \\
\hline
\end{tabular}

t-Test mit ungleichen Varianzen

\section{Varianzanalysen}

- Die Angabe von regelmäßigem Alkoholkonsum hat einen signifikanten Einfluss auf schlechtere Schlafqualität ( $p=0,003)$.

- Das Alter, das Geschlecht, die Dauer der Abhängigkeit und die Dauer der Substitution haben keinen Einfluss auf die Schlafqualität.

- Zusammenhänge zwischen der Höhe der Dosis und dem PSQIGlobalscore wurden über Pearsons r überprüft. Es ergaben sich für die Methadongruppe $(r=0,193, p=<0,01)$, die Levomethadongruppe $(r=0,294, p=<0,01)$ und für die Buprenorphingruppe $(r=0,333, p=0,013)$ leicht positive Korrelationen.

\section{Auswertung nach beiverordneten Medikamenten}

Die Analyse der Substitutionsgruppen nach beiverordneten Medikamenten ergab signifikant schlechtere Schlafqualitäten in allen Gruppen mit Ausnahme der Patienten unter Neuroleptika (Tab. 6).

\section{Diskussion}

Die hier vorliegende Untersuchung wurde als anonymisierte Studie geplant, um ein möglichst großes Patientenkollektiv zu gewinnen. Daher wurde auf detaillierte Fragen zur psychosozialen Situation verzichtet. Die Tatsache, dass einige Patienten Angaben zu Alter, Dosierung und Abhängigkeitsdauer verweigert haben, zeigt die Richtigkeit dieser Entscheidung. Gewisse Selektionsmechanismen und Verzerrungen durch Drittvariable sind daher nicht auszuschließen.
Tab. 6 PSQI-Globalscore nach beiverordneten Medikamenten

\begin{tabular}{|lllll}
\hline Medikamente & mean & SD & p & n \\
\hline keine & 8,7 & 4,6 & & 371 \\
\hline Benzodiazepine & 11,6 & 5 & $<0,001$ & 43 \\
\hline Antidepressiva & 10,8 & 4,2 & 0,001 & 57 \\
\hline Neuroleptika & 10 & 6 & n. s. & 10 \\
\hline sonstige & 10,7 & 5 & $<0,001$ & 95 \\
\hline
\end{tabular}

Alle Substitutionsgruppen mit Ausnahme der DHC-Gruppe weisen eine signifikant schlechtere Schlafqualität im Vergleich $\mathrm{zu}$ den Kontrollen auf. Dies entspricht zumindest bei Methadon und Levomethadon den Erfahrungen in unserer täglichen Praxis.

Buprenorphinpatienten erreichen einen niedrigeren PSQI-Globalscore im Vergleich zur Methadon/Levomethadongruppe, ohne dass der Unterschied Signifikanz erreicht, es wird allerdings auch kein signifikanter Unterschied zur Naltrexongruppe erreicht. Dies kann als Hinweis darauf gedeutet werden, dass Buprenorphin bez. der hier untersuchten Parameter eine Tendenz zu einem geringeren Nebenwirkungsspektrum aufweist. Auffällig ist das „gute“ Abschneiden der DHC-Patienten, die keine wesentlichen Differenzen zur Kontroll- und Naltrexongruppe aufweisen, sich aber mit signifikant niedrigeren Scores von der Methadon- und Levomethadongruppe abheben. Hier besteht ein dringender Bedarf für eine prospektive Studie. Es könnte sich eine interessante Therapieoption für schlafgestörte Substitutionspatienten ergeben. 
Die Naltrexongruppe weist, wie zu erwarten, keine signifikanten Differenzen zur Kontrollgruppe auf.

Betrachtet man die Durchschlafstörungen, auf die in Frage 5b des PSQI Bezug genommen wird, ergibt sich ein ähnliches Bild. Sämtliche Substitutionsgruppen weisen einen signifikant höheren Score im Vergleich zur Kontroll- und zur Naltrexongruppe auf. Auch hier zeigen die DHC-Patienten eine Tendenz zu niedrigeren Scores, ohne jedoch Signifikanz zu erreichen. Ein schwer interpretierbares Bild zeigt sich in Bezug auf Schlafstörungen durch nächtliches Wasserlassen. Der hochsignifikante Unterschied aller Substitutionsgruppen gegen die Kontrollgruppe ist überraschend. Bekanntermaßen erhöht Morphin den Tonus des Ureters und hemmt die Blasenentleerung. Es entwickelt sich eine Toleranz gegenüber diesen Effekten. Die hier erhobenen Befunde sind dem diametral entgegengesetzt. Dieses Phänomen ist als klinisch beobachtete Nebenwirkung nicht bekannt [28]. Eine mögliche spekulative Erklärung besteht in der Annahme, dass eine Schlafstörung als primäres Ereignis imponiert und eine teilweise gefüllte Blase beim nächtlichen Erwachen als auslösende Ursache zu Unrecht angeschuldigt wird.

Zusätzlich muss Alkoholkonsum vor dem Schlafengehen als mögliche Ursache erwogen werden. Ein entsprechender Einfluss konnte nachgewiesen werden.

Betrachtet man den Anteil schwerer Schlafstörungen, definiert als PSQI-Globalscore $>10$, ergibt sich ein identisches Bild wie bei der Analyse des PSQI-Globalscores. Die Methadon-, Levomethadon- und Buprenorphingruppe weisen einen signifikant höheren Anteil von Patienten mit schweren Schlafstörungen im Vergleich zur Kontrollgruppe auf. Die Differenzen bei Naltrexon und DHC erreichen keine Signifikanz.

Die Ergebnisse des ESS scores, die keine signifikanten Unterschiede zwischen den Gruppen erbrachten, stehen in Einklang mit der Literatur, wonach Insomniepatienten keine erhöhte Tagesschläfrigkeit aufweisen $[3,4,6]$. Im Gegensatz zur Literatur $[25,26]$ konnte in dieser Studie lediglich in der dl-Methadongruppe ein Einfluss von Durchschlafstörungen auf die Tagesschläfrigkeit gefunden werden. Die Schlafeffektivität hatte keinen Einfluss auf die Schlafqualität.

Die Varianzanalysen erbrachten einen erwarteten Einfluss von Alkoholkonsum auf die Schlafqualität, alle anderen erhobenen Parameter zeigten keinen Einfluss. Allerdings bestand in der Methadon-, Levomethadon- und Buprenorphingruppe eine signifikant positive Korrelation zwischen PSQI-Globalscore und der Medikamentendosis. Dies kann zweierlei bedeuten: Zum ersten ist anzunehmen, dass Hochdosispatienten instabiler sind und daher auch eher zu Schlafstörungen neigen, zum anderen kann ein Medikamenteneffekt, der mit steigender Dosis zunimmt, für dieses Phänomen verantwortlich sein, entsprechende Literaturhinweise liegen vor [20].

Die Ergebnisse der Auswertung des PSQI-Globalscores nach beiverordneten Medikamenten waren zu erwarten. Es darf unterstellt werden, dass ein wesentlicher Teil der Patienten unter Antidepressiva oder Benzodiazepinen diese eben wegen ihrer Schlafstörungen bzw. wegen einer depressiven Störung, die gleichfalls häufig mit Schlafstörungen einhergeht, verordnet bekamen. Die weiterhin schlechte Schlafqualität macht aber den Bedarf nach effektiveren Behandlungsstrategien deutlich.

Die über alle Parameter in sich konsistenten Ergebnisse dieser Studie sowie der Nachweis einer Korrelation zwischen Medikamentendosis und PSQI-Globalscore lassen bei aller Zurückhaltung psychosoziale Faktoren nicht als alleinige Ursachen für eingeschränkte Schlafqualität erscheinen. Es müssen unbedingt pharmakologische Ursachen für gestörten Schlaf unter Opiattherapie bedacht werden, wie sie in der Literatur beschrieben werden (s. Tab.1). Es besteht dringender Bedarf an kontrollierten prospektiven Studien zu diesem Thema, hierbei sollten auch die auffälligen Ergebnisse für DHC Eingang finden.

\section{Literatur}

${ }^{1}$ Frankel BL, Coursey RD, Buchbinder R et al. Recorded and reported sleep in chronic primary insomnia. Archives of General Psychiatry 1976; 133: $615-623$

${ }^{2}$ Carskadon MA, Dement WC, Mitler MM et al. Self-reports versus sleep laboratory findings in 122 drug-free subjects with complaints of chronic insomnia. Archives of General Psychiatry 1976; 133: 1382 - 1388

${ }^{3}$ Johns MW. A new method for measuring daytime sleepiness: the Epworth sleepiness scale. Sleep 1991; 14 (6): 540-545

${ }^{4}$ Chervin RD, Aldrich MS, Pickett R et al. Comparison of the results of the Epworth Sleepiness Scale and the Multiple Sleep Latency Test. J Psychosom Res 1997; 42 (2): 145-155

5 Johns MW. Reliability and factor analysis of the Epworth Sleepiness Scale. Sleep 1992; 15 (4): 376 - 381

${ }^{6}$ Olson LG, Cole MF, Ambrogetti A. Correlations among Epworth Sleepiness Scale scores, multiple sleep latency tests and psychological symptoms. J-Sleep-Res 1998; 7(4): 248-253

${ }^{7}$ Hohagen F, Rink K, Käppler C et al. Prevalence and treatment of insomnia in general practice. A longitudinal study. Eur. Arch Psychiatry Clin Neurosci 1993; 242: 329-336

${ }^{8}$ Wittchen HU, Krause P, Höfler M et al. NISAS-2000: Die „Nationwide Insomnia screening and awareness Study“. Fortschritte der Medizin 2001; 119: Originalien Nr.1/2001

${ }^{9}$ Simen S, Hajak G, Schlaf G et al. Chronifizierung von Schlafbeschwerden. Ergebnisse einer Repräsentativumfrage in Westdeutschland. Nervenarzt 1995; 66: 686-695

10 Oyefeso A, Sedwick P, Ghodse H. Subjective sleep-wake parameters in treatment-seeking opiate addicts. Drug and Alcohol dependence 1997; 48: 9-16

${ }^{11}$ Stein MB, Chartier M, Walker JR. Sleep in nondepressed patients with panic disorder: I. Systematic assessment of subjective sleep quality and sleep disturbance. Sleep 2001; 16: 724-746

${ }^{12}$ Steinberg R. Schlafmedizin - Grundlagen und Praxis Bremen. UNIMED 2000

${ }^{13}$ Gann H, Landbrecht M, Feige B et al. Subjektive Parameter des Schlafes bei Patienten mit primärer Alkoholabhängigkeit. Somnologie 2001; 5: $65-70$

${ }^{14}$ Laudon $\mathrm{M}$ et al. Melatonin effectively facilitates benzodiazepine discontinuation in insomnia patients. Abstract of the third international congress of the world federation of sleep research societies. Dresden 1999

${ }^{15}$ Norman SE, Chediak AD, Freeman C et al. Sleep disturbances in men with asymptomatic human immunodeficiency (HIV) Infektion. Sleep 2001; 15: 150-155

${ }^{16}$ Snyder EW, Dustman RE, Beck EC. Sustained ingestion of methadone and the sleep of monkeys. Psychopharmacology (Berlin) 1978; 60: $29-34$

17 Staedt J, Wassmuth F, Stoppe G et al. Effects of chronic treatment with methadone and naltrexone on sleep in addicts. European Archives of Psychiatry Clinical Neurscience 1996; 246: 305 - 309

${ }^{18}$ Kay DC. Human sleep during chronic morphine intoxikation. Psychopharmacologia 1975 ; 44: $117-124$ 
${ }^{19}$ Teichtahl H, Prodromidis A, Miller B et al. Sleep-disordered breathing in stable methadone programme patients: a pilot study. Addiction 2001; 96: 395-403

${ }^{20}$ Kay DC, Pickworth WB, Neidert GL et al. Opioid effects on computedderived sleep and EEG parameters in nondependent human addicts. Sleep 1979; 2: 175 - 191

${ }^{21}$ Pickworth WB, Neidert GL, Kay DC. Morphinelike arousal by methadone during sleep. Clinical Pharmacology and therapeutics 1981; 30: $796-804$

${ }^{22}$ Hatzinger M, Kocher R, Hemmeter U et al. Schlaf und Sucht (Sleep and addiction). Rundschau für med Praxis. Schweiz 1995; 84: 450-453

${ }^{23}$ Lewis SA, Oswald I, Evans JI et al. Heroin and human sleep. Electroencephalog Clin Neurophysiol 1970; 28: $374-381$

${ }^{24}$ Orr WC, Stahl ML. Sleep patterns in human methadone addiction. British Journal of addiction to alcohol and other drugs 1978; 73: 311 - 315

${ }^{25}$ Martin S, Engleman H, Deary I, Douglas N. The effect on sleep fragmentation on daytime dysfunction. American Journal of respiratory and critical care Medicine 1996; 153: $1328-1332$

${ }^{26}$ Stepanski E, Lampshire J, Badia P et al. Sleep fragmentation and daytime sleepiness. Sleep 1984; 7: 18-26

27 Buysse DJ, Reynolds CF, Monk TH et al. The Pittsburgh Sleep Quality Index: A new instrument for psychiatric practice and research. Psychiatry Research 1989; 28: $193-213$

${ }^{28}$ Fuhr U. Persönliche Mitteilung. 2002

\begin{tabular}{ll}
\hline Arbter, P. A. & Krefeld \\
\hline Bonus, S. & Köln \\
\hline Buchmann-Pehlgrimm, U. & Euskirchen \\
\hline Böhm, M. & Berlin \\
\hline Brack, M. & Urspringen \\
\hline Beteiligte Zentren/Praxen & \\
\hline Busch, H. & Münster \\
\hline Geisler, U. & Hagen \\
\hline Gölz, J. & Berlin \\
\hline Gospodinov, B. & Saarbrücken \\
\hline Gülick van, B. & Leverkusen \\
\hline Hander, E. & Duisburg \\
\hline Hoenekopp, I. & Mannheim \\
\hline Isernhagen, K. & Köln \\
\hline Krolewski, R. & Gummersbach \\
\hline Leuchtmann, H. & Suchtambulanz, LKH Köln-Merheim \\
\hline Pieper, J. & Bonn \\
\hline Poehlke, T. & Münster \\
\hline Sartoris, R. & Köln \\
\hline Sauer, G. & Reichelsheim \\
\hline Schnaitmann, E. & Stuttgart \\
\hline Schölzel, S. & Troisdorf \\
\hline Schroeder, E. & Bremen \\
\hline Schuler-Kuon, B. & Leutkirch \\
\hline Siepe, W. & Köln \\
\hline Steininger, R. & Köln \\
\hline Stolberg, H. & Eschweiler \\
\hline Sworszt, S. & Gelsenkirchen \\
\hline Ullmann, R. & Hamburg \\
\hline Ulmer, A. & Stuttgart \\
\hline Voss, M. & Köln \\
\hline Wagner, J. & Ludwigshafen \\
\hline Walter, H. & Hamm \\
\hline Weckbecker, K. & Bad Honnef \\
\hline
\end{tabular}

